\title{
On Bernstein-Type Theorems in Semi-Riemannian Warped Products
}

\author{
Wenjie Wang ${ }^{1}$ and Ximin Liu ${ }^{2}$ \\ ${ }^{1}$ Department of Mathematics, South China University of Technology, Guangzhou, Guangdong 510641, China \\ ${ }^{2}$ School of Mathematical Sciences, Dalian University of Technology, Dalian, Liaoning 116024, China \\ Correspondence should be addressed to Wenjie Wang; wangwj072@163.com
}

Received 10 April 2013; Revised 29 July 2013; Accepted 30 July 2013

Academic Editor: Shao-Ming Fei

Copyright (c) 2013 W. Wang and X. Liu. This is an open access article distributed under the Creative Commons Attribution License, which permits unrestricted use, distribution, and reproduction in any medium, provided the original work is properly cited.

Complete spacelike hypersurfaces immersed in semi-Riemannian warped products are investigated. By using a technique according to Yau (1976) and a reasonable restriction on the mean curvature of the hypersurfaces, we obtain some new Bernstein-type theorems which extend some known results proved by Camargo et al. (2011) and Colares and Lima (2012).

\section{Introduction}

In the present paper, we are interested in the study of the complete spacelike hypersurfaces immersed in semi-Riemannian warped product manifolds, in particular, in steady state-type spacetime and hyperbolic-type space. Before giving details of our main results, we first present a brief outline of some recent papers containing theorems related to ours.

By using a restriction on the height function of a complete spacelike hypersurface, Caminha and de Lima [1] obtained some unique results concerning complete spacelike hypersurfaces with constant mean curvature immersed in steady state space and hyperbolic space, respectively.

Later, Albujer and Alías [2] proved that on a complete spacelike hypersurface the constant mean curvature must be identically 1 , provided that such a hypersurface is bounded away from the infinity of the steady state space. For some other Bernstein-type results concerning constant mean curvature, we refer the reader to some recent papers by Albujer et al. [3, 4] and Aquino and de Lima [5].

Also, by using the well known result according to Yau [6], Camargo et al. in [7] obtained some Bernstein-type results concerning complete spacelike hypersurfaces, in steady statetype and hyperbolic-type space. Noticing that in their paper the mean curvature of the complete spacelike hypersurface need not be a constant. de Lima in [8] obtained a new Bernstein-type theorem concerning complete spacelike hypersurfaces in hyperbolic space with the bounded mean curvature (not necessarily constant) and a restriction on the normal angle.

In this paper, following $[9,10]$ we shall consider the Laplacian of the integral of the warping function. In fact, by using a technique provided by Yau in [6] and supposing an appropriate restriction on the mean curvature, we obtain the following Bernstein-type theorems.

Theorem 1. Let $\bar{M}^{n+1}=-I \times{ }_{f} M^{n}$ be a generalized RobertsonWalker spacetime whose fiber $M^{n}$ is a complete Riemannian manifold. Let $\psi: \Sigma^{n} \rightarrow \bar{M}^{n+1}$ be a complete and connected spacelike hypersurface with the mean curvature $H$ satisfying

$$
H \geq \sup _{\Sigma^{n}} \frac{f^{\prime}}{f}(h)>0 .
$$

If $\nabla h$ has integrable norm on $\Sigma^{n}$, then $\Sigma^{n}$ is a slice of $-I \times{ }_{f} M^{n}$.

The Riemannian version of Theorem 1 is also presented as follows.

Theorem 2. Let $\bar{M}^{n+1}=I \times{ }_{f} M^{n}$ be a Riemannian warped product whose fiber $M^{n}$ is a complete Riemannian manifold. 
Let $\psi: \Sigma^{n} \rightarrow \bar{M}^{n+1}$ be a complete and connected spacelike hypersurface with the mean curvature $H$ satisfying

$$
0<H \leq \inf _{\Sigma^{n}} \frac{f^{\prime}}{f}(h) .
$$

If $\nabla h$ has integrable norm on $\Sigma^{n}$, then $\Sigma^{n}$ is a slice of $I \times_{f} M^{n}$.

Suppose that the warping function $f$ in both Theorems 1 and 2 is given by $f=e^{t}$ for $t \in I$, then the above two theorems are just the corresponding results shown in [7]. Finally, letting the warping function $f$ in Theorem 2 be $f=e^{t}$ for $t \in \mathbb{R}$ and the fiber $M^{n}$ an $n$-dimensional Euclidean space, then Theorem 2 gives a Bernstein-type theorem for spacelike hypersurfaces immersed in the hyperbolic space (see Section 4 for details).

This paper is organized as follows. We shall first recall some notations and collect some basic facts in a preliminaries section, and some key lemmas used to prove our main theorems are also given in this section. Section 3 is devoted to proving some unique theorems concerning spacelike hypersurfaces in semi-Riemannian warped products. Finally, in Section 4, some applications of our main theorems in steady state-type spacetimes and hyperbolic-type spaces are obtained respectively.

\section{Preliminaries}

In this section, from [11, 12] we shall recall some basic notations and facts that will appear along this paper.

Let $M^{n}$ be a connected, $n$-dimensional $(n \geq 2)$ oriented Riemannian manifold, $I \subseteq \mathbb{R}$ an interval, and $f: I \rightarrow$ $\mathbb{R}$ a positive smooth function. We consider the product differential manifold $I \times M^{n}$ and denote by $\pi_{I}$ and $\pi_{M}$ the projections onto the base $I$ and fiber $M^{n}$, respectively. A particular class of semi-Riemannian manifolds is the one obtained by furnishing product manifolds $I \times M^{n}$ with the metric

$$
\begin{aligned}
\langle v, w\rangle_{p}= & \epsilon\left\langle\left(\pi_{I}\right)_{*} v,\left(\pi_{I}\right)_{*} w\right\rangle+\left(f \circ \pi_{I}(p)\right)^{2} \\
& \times\left\langle\left(\pi_{M}\right)_{*} v,\left(\pi_{M}\right)_{*} w\right\rangle,
\end{aligned}
$$

for any $p \in \bar{M}^{n+1}$ and any $v, w \in T_{p} \bar{M}^{n+1}$, where $\epsilon= \pm 1$. We call such a space warped product manifold, and $f$ is known as the warping function and we denote the space by $\bar{M}^{n+1}=\epsilon I \times_{f} M^{n}$. Note that $-I \times_{f} M^{n}$ is called a generalized Robertson-Walker spacetime [11], in particular, $-I \times{ }_{f} M^{n}$ is called a Robertson-Walker spacetime if the fiber $M^{n}$ has constant sectional curvature. From [13] we know that a generalized Robertson-Walker spacetime has constant sectional curvature $\bar{k}$ if and only if the Riemannian fiber $M^{n}$ has constant sectional curvature $k$ and the warping function $f$ satisfies the following differential equation:

$$
\frac{f^{\prime \prime}}{f}=\bar{k}=\frac{f^{\prime 2}+k}{f^{2}} .
$$

It follows from $[14,15]$ that the vector field $\left(f \circ \pi_{I}\right) \partial_{t}$ is conformal and closed (in this sense that its dual 1-form is closed) with conformal factor $\phi=f^{\prime} \circ \pi_{I}$, where the prime denotes differentiation with respect to $t \in I$. For $t_{0} \in I$, we orient the slice $\Sigma_{t_{0}}^{n}:=\left\{t_{0}\right\} \times M^{n}$ by the unit normal vector field $\partial_{t}$, then from [9] we know that $\sum_{t_{0}}^{n}$ has constant $r$ th mean curvature $H_{r}=-\epsilon\left(f^{\prime}\left(t_{0}\right) / f\left(t_{0}\right)\right)^{r}$ with respect to $\partial_{t}$ for $0 \leq r \leq n$.

A smooth immersion $\psi: \Sigma^{n} \rightarrow \epsilon I \times_{f} M^{n}$ of an $n$-dimensional connected manifold $\Sigma^{n}$ is said to be a spacelike hypersurface if the induced metric via $\psi$ is a Riemannian metric on $\Sigma^{n}$. If $\Sigma^{n}$ is oriented by the unit vector field $N$, one obviously has $\epsilon=\epsilon_{\partial_{t}}=\epsilon_{N}$.

We consider two particular functions naturally attached to complete spacelike hypersurfaces, namely, the vertical (height) function $h=\left.\left(\pi_{I}\right)\right|_{\Sigma^{n}}$ and the support function $\left\langle N, \partial_{t}\right\rangle$. We denote by $\bar{\nabla}$ and $\nabla$ the gradients with respect to the metrics of $\epsilon I \times{ }_{f} M^{n}$ and $\Sigma^{n}$, respectively. Thus, by a simple computation we present the gradient of $\pi_{I}$ on $\epsilon I \times{ }_{f} M^{n}$ as follows:

$$
\bar{\nabla} \pi_{I}=\epsilon\left\langle\bar{\nabla} \pi_{I}, \partial_{t}\right\rangle \partial_{t}=\epsilon \partial_{t} .
$$

Moreover, the gradient of $h$ on $\Sigma^{n}$ is given by

$$
\nabla h=\left(\bar{\nabla} \pi_{I}\right)^{\top}=\epsilon\left(\partial_{t}\right)^{\top}=\epsilon \partial_{t}-\left\langle N, \partial_{t}\right\rangle N .
$$

We denote by $|\cdot|$ the norm of a vector field on $\Sigma^{n}$, then we get

$$
|\nabla h|^{2}=\epsilon\left(1-\left\langle N, \partial_{t}\right\rangle^{2}\right) .
$$

According to $[2,16]$, a spacelike hypersurface $\psi: \Sigma^{n} \rightarrow$ $\epsilon I \times{ }_{f} M^{n}$ is said to be bounded away from the future infinity of $\epsilon I \times{ }_{f} M^{n}$ if there exists $\bar{t} \in I$ such that

$$
\psi\left(\Sigma^{n}\right) \subset\left\{(t, p) \in \epsilon I \times{ }_{f} M^{n}: t \leq \bar{t}\right\} .
$$

Analogously, a spacelike hypersurface $\psi: \Sigma^{n} \rightarrow \epsilon I \times_{f} M^{n}$ is said to be bounded away from the past infinity of $\epsilon I \times{ }_{f} M^{n}$ if there exists $\underline{t} \in I$ such that

$$
\psi\left(\Sigma^{n}\right) \subset\left\{(t, p) \in \epsilon I \times{ }_{f} M^{n}: t \geq \underline{t}\right\} .
$$

Finally, $\Sigma^{n}$ is said to be bounded away from the infinity of $\epsilon I \times{ }_{f} M^{n}$ if it is both bounded away from the past and future infinity of $\epsilon I \times{ }_{f} M^{n}$.

Setting $k=0$ in Lemma 4.1 of [9], we may obtain the Laplacian of the integral of the warping function in a generalized Robertson-Walker spacetime. By using the technique according to Alías and Colares [9], the second author and Wang in [10] generalize this result in a semiRiemannian warped product as follows.

Lemma 3 (see $[9,10])$. Let $\psi: \Sigma^{n} \rightarrow \epsilon I \times{ }_{f} M^{n}$ be a spacelike hypersurface immersed in a semi-Riemannian warped product. If

$$
\sigma(t)=\int_{t_{0}}^{t} f(s) d s
$$


then

$$
\Delta \sigma(h)=\epsilon n\left(f^{\prime}(h)+f(h)\left\langle N, \partial_{t}\right\rangle H\right),
$$

where $\Delta$ denotes the Laplacian operator and $h$ is the height function of $\Sigma^{n}$.

Furthermore, we also need the following well known lemma according to Yau [6].

Lemma 4 (corollary on page 660 of [6]). Let $\Sigma^{n}$ be an $n$ dimensional complete Riemannian manifold. If $g: \Sigma^{n} \rightarrow \mathbb{R}$ is a smooth subharmonic or superharmonic function whose gradient norm is integrable on $\Sigma^{n}$, then $g$ must be actually harmonic.

\section{Proofs of Main Theorems}

Based on the above arguments in Section 2, we may present the proof of Theorem 1 as follows.

Proof of Theorem 1. Since $\partial_{t}$ is a unitary timelike vector field globally defined on the ambient spacetime, then there exists a unique timelike unitary normal vector filed $N$ globally defined on the spacelike hypersurface $\Sigma^{n}$ which is the same time orientation as $\partial_{t}$. By using the reverse Cauchy-Schwarz inequality we have

$$
\left.\left\langle N, \partial_{t}\right\rangle\right|_{\Sigma^{n}} \leq-1<0 .
$$

Letting $\epsilon=-1$, then it follows from Lemma 3 that

$$
\Delta \sigma(h)=-n\left(f^{\prime}(h)+f(h)\left\langle N, \partial_{t}\right\rangle H\right) .
$$

The assumptions of Theorem 1 assure that $H>0$, then, it follows from (12) that

$$
\left\langle N, \partial_{t}\right\rangle H \leq-H<0 .
$$

Noticing that the warping function is positive on $I$, then, by using the above inequality and (1) in (13) we have the following inequality:

$$
\begin{aligned}
\Delta \sigma(h) & =-n f(h)\left(\frac{f^{\prime}(h)}{f(h)}+\left\langle N, \partial_{t}\right\rangle H\right) \\
& \geq-n f(h)\left(\frac{f^{\prime}(h)}{f(h)}-H\right) \\
& \geq-n f(h)\left(\sup _{\Sigma^{n}} \frac{f^{\prime}(h)}{f(h)}-H\right) \geq 0,
\end{aligned}
$$

this means that $\sigma(h)$ is a subharmonic function on $\Sigma^{n}$.

On the other hand, since the spacelike hypersurface $\Sigma^{n}$ is bounded away from the infinity of $-I \times{ }_{f} M^{n}$, then the height function $h$ is bounded on $\Sigma^{n}$. Also, we have

$$
|\nabla \sigma(h)|=f(h)|\nabla h| .
$$

Since $|\nabla h|$ is integrable on $\Sigma^{n}$, from (16) we know that $|\nabla \sigma(h)|$ is also integrable on $\Sigma^{n}$. The above arguments assure that Lemma 4 is applicable, then applying Lemma 4 on subharmonic function $\sigma(h)$ on $\Sigma^{n}$ we have

$$
\Delta \sigma(h)=0 .
$$

Putting the above equation into (15) and noting that the warping function $f$ is a smooth positive function on $I$, we obtain $\left(f^{\prime} / f\right)(h)=-\left\langle N, \partial_{t}\right\rangle H$, thus, using inequalities (1) and (12) in this equation we have

$$
\sup _{\Sigma^{n}} \frac{f^{\prime}}{f}(h) \geq \frac{f^{\prime}}{f}(h)=-\left\langle N, \partial_{t}\right\rangle H \geq-\left\langle N, \partial_{t}\right\rangle \sup _{\Sigma^{n}} \frac{f^{\prime}}{f}(h) .
$$

The hypothesis (1) implies that $\sup _{\Sigma^{n}}\left(f^{\prime} / f\right)(h)>0$ on $\Sigma^{n}$, then it follows from the above inequality that $-\left\langle N, \partial_{t}\right\rangle \leq 1$; comparing this inequality with inequality (12) we obtain an identity $\left\langle N, \partial_{t}\right\rangle=-1$. Finally, using $\epsilon=-1$ and $\left\langle N, \partial_{t}\right\rangle=-1$ in (7) gives that

$$
|\nabla h|^{2}=-\left(1-\left\langle N, \partial_{t}\right\rangle^{2}\right) \equiv 0,
$$

which means that $h$ is a constant on $\Sigma^{n}$. Then we prove that $\Sigma^{n}$ is a slice of $-I \times{ }_{f} M^{n}$.

Next we give the Riemannian version of the proof of Theorem 1 on a Riemannian warped product space as follows.

Proof of Theorem 2. In this context, we may consider $N$ being the orientation of the hypersurface $\Sigma^{n}$ such that its angle function satisfies

$$
-1 \leq\left.\left\langle N, \partial_{t}\right\rangle\right|_{\Sigma^{n}} \leq 0 .
$$

Now, letting $\epsilon=1$, then it follows from Lemma 3 that

$$
\Delta \sigma(h)=n\left(f^{\prime}(h)+f(h)\left\langle N, \partial_{t}\right\rangle H\right) .
$$

The assumption (2) implies that $H$ is positive, then, from (20) we have that

$$
-H \leq\left\langle N, \partial_{t}\right\rangle H \leq 0 .
$$

Noticing that the warping function is positive on $I$, then, using the above inequality and (2) in (21) we obtain

$$
\begin{aligned}
\Delta \sigma(h) & =n f(h)\left(\frac{f^{\prime}(h)}{f(h)}+\left\langle N, \partial_{t}\right\rangle H\right) \\
& \geq n f(h)\left(\frac{f^{\prime}(h)}{f(h)}-H\right) \\
& \geq n f(h)\left(\inf _{\Sigma^{n}} \frac{f^{\prime}(h)}{f(h)}-H\right) \geq 0,
\end{aligned}
$$

this means that $\sigma(h)$ is a subharmonic function on $\Sigma^{n}$.

As the spacelike hypersurface $\Sigma^{n}$ is bounded away from the infinity of $I \times{ }_{f} M^{n}$, then the height function $h$ is also bounded on $\Sigma^{n}$. Also, (16) holds in this context. As $|\nabla h|$ is integrable on $\Sigma^{n}$, then (16) assures that $|\nabla \sigma(h)|$ is also 
integrable. From the above arguments we see that Lemma 4 is applicable; applying Lemma 4 on subharmonic function $\sigma(h)$ we obtain (17). Thus, putting (17) into (23) and noting that the warping function $f$ is positive on $I$, we obtain $\left(f^{\prime} / f\right)(h)=$ $-\left\langle N, \partial_{t}\right\rangle H$, using inequality (2) in this equation yields that

$$
\begin{aligned}
0 & <\inf _{\Sigma^{n}} \frac{f^{\prime}}{f}(h) \leq \frac{f^{\prime}}{f}(h)=-\left\langle N, \partial_{t}\right\rangle H \\
& \leq-\left\langle N, \partial_{t}\right\rangle \inf _{\Sigma^{n}} \frac{f^{\prime}}{f}(h) .
\end{aligned}
$$

It follows from (24) that $-\left\langle N, \partial_{t}\right\rangle \geq 1$, comparing this inequality with inequality (20) we obtain identity $\left\langle N, \partial_{t}\right\rangle=$ -1 . Finally, using $\epsilon=1$ and $\left\langle N, \partial_{t}\right\rangle=-1$ in (7) gives

$$
|\nabla h|^{2}=1-\left\langle N, \partial_{t}\right\rangle^{2} \equiv 0,
$$

which means that $h$ is a constant on $\Sigma^{n}$. Then we prove that $\Sigma^{n}$ is a slice of $I \times{ }_{f} M^{n}$.

Remark 5. It is worth to point out that Colares and de Lima [17] obtained some rigidity theorems in semi-Riemannian warped products which are similar to ours. However, in the assumptions of Theorems 4.2 and 4.6 of [17], the warping function $f$ is assumed to has convex logarithm. On the other hand, we refer the reader to $[12,15]$ for some examples of semi-Riemannian warped products whose warping functions are not necessarily to have convex logarithm. That is, without requiring the assumption that $\ln f$ is convex, our Theorems 1 and 2 attain the same conclusions as the corresponding results proved in [17].

\section{Applications}

In this section, we apply our main theorems on some physical models, in particular, on steady state-type space spacetime and hyperbolic-type space.

According to [2], $-\mathbb{R} \times{ }_{e^{t}} M^{n}$ is said to be a steady statetype spacetime, where $M^{n}$ is an $n$-dimensional complete and connected Riemannian manifold. In particular, $-\mathbb{R} \times e_{e^{t}} \mathbb{R}^{n}$ is called the $(n+1)$-dimensional steady state spacetime, which is isometric to an open subset of the de Sitter space $\mathbb{S}^{n+1}$. The importance of studying the steady state spacetime comes from the fact that, in cosmology, $-\mathbb{R} \times e_{e^{t}} \mathbb{R}^{3}$ is the steady state model of the universe proposed by Bondi and Gold [18], and Hoyle [19].

Suppose that the warping function is given by $f=e^{t}$, then the following result follows from Theorem 1 .

Corollary 6. Let $\psi: \Sigma^{n} \rightarrow-\mathbb{R} \times{ }_{e^{t}} M^{n}$ be a complete spacelike hypersurface in steady state-type spacetime. Suppose that $\Sigma^{n}$ is bounded away from the infinity of $-\mathbb{R} \times{ }_{e^{t}} M^{n}$ and that the mean curvature $H$ satisfies $H \geq 1$. If $\nabla$ h has integrable norm on $\Sigma^{n}$, then $H=1$ and $\Sigma^{n}$ is a slice of $-\mathbb{R} \times{ }_{f} M^{n}$.

We remark that Corollary 6 was proved in [7] by using a different method from ours.
The hyperbolic-type space is defined by $\mathbb{R} \times{ }_{e^{t}} M^{n}$, where $M^{n}$ is a complete connected Riemannian manifold. The motivation for investigating the hyperbolic-type space $\mathbb{R} \times{ }_{e^{t}} M^{n}$ comes from the fact that the $(n+1)$-dimensional hyperbolic space $\mathbb{U}^{n+1}$ is isometric to $\mathbb{R} \times e_{e^{t}} \mathbb{R}^{n}$. Noting that an explicit isometry between the half-space model and this hyperbolictype model has been pointed out by Alías and Dajczer in [20].

Now letting the warping function be $f=e^{t}$ for $t \in \mathbb{R}$, then the following result follows from Theorem 2 .

Corollary 7. Let $\psi: \sum^{n} \rightarrow \mathbb{R} \times{ }_{e^{t}} M^{n}$ be a complete spacelike hypersurface in hyperbolic-type space. Suppose that $\sum^{n}$ is bounded away from the infinity of $\mathbb{R} \times{ }_{e^{t}} M^{n}$ and that the mean curvature $H$ satisfies $0<H \leq 1$. If $\nabla h$ has integrable norm on $\Sigma^{n}$, then $H=1$ and $\Sigma^{n}$ is a slice of $\mathbb{R} \times{ }_{e^{t}} M^{n}$.

Also, letting the warping function be $f=e^{t}$ and $M^{n}$ an $n$-dimensional Euclidean space, then the following result follows from Corollary 7.

Corollary 8. Let $\psi: \Sigma^{n} \rightarrow \mathbb{R} \times_{e^{t}} \mathbb{R}^{n}$ be a complete spacelike hypersurface in a hyperbolic space. Suppose that $\Sigma^{n}$ is bounded away from the infinity of $\mathbb{R} \times e_{e^{t}} \mathbb{R}^{n}$ and that the mean curvature $H$ satisfies $0<H \leq 1$. If $\nabla h$ has integrable norm on $\Sigma^{n}$, then $H=1$ and $\Sigma^{n}$ is a horosphere of $\mathbb{R} \times \times_{e^{t}} \mathbb{R}^{n}$.

Remark 9. De Lima in [8] proved that a complete spacelike hypersurface immersed in hyperbolic space with the mean curvature $0 \leq H \leq 1$ and $-\left\langle N, \partial_{t}\right\rangle \geq \sup _{\Sigma^{n}} H$ is a horosphere, provided that $\Sigma^{n}$ is under a horosphere of the hyperbolic space and the second fundamental from is bounded.

\section{Acknowledgment}

The project is supported by the Natural Science Foundation of China (no. 10931005) and the Natural Science Foundation of Guangdong Province of China (no. S2011010000471).

\section{References}

[1] A. Caminha and H. F. de Lima, "Complete vertical graphs with constant mean curvature in semi-Riemannian warped products," Bulletin of the Belgian Mathematical Society. Simon Stevin, vol. 16, no. 1, pp. 91-105, 2009.

[2] A. L. Albujer and L. J. Alías, "Spacelike hypersurfaces with constant mean curvature in the steady state space," Proceedings of the American Mathematical Society, vol. 137, no. 2, pp. 711-721, 2009.

[3] A. L. Albujer, F. E. C. Camargo, and H. F. de Lima, "Complete spacelike hypersurfaces with constant mean curvature in $-\mathbb{R} \times$ $\mathbb{U}^{n}$, , Journal of Mathematical Analysis and Applications, vol. 368, no. 2, pp. 650-657, 2010.

[4] A. L. Albujer, F. E. C. Camargo, and H. F. de Lima, "Complete spacelike hypersurfaces in a Robertson-Walker spacetime," Mathematical Proceedings of the Cambridge Philosophical Society, vol. 151, no. 2, pp. 271-282, 2011.

[5] C. P. Aquino and H. F. de Lima, "On the rigidity of constant mean curvature complete vertical graphs in warped products," Differential Geometry and its Applications, vol. 29, no. 4, pp. 590-596, 2011. 
[6] S. T. Yau, "Some function-theoretic properties of complete Riemannian manifold and their applications to geometry," Indiana University Mathematics Journal, vol. 25, no. 7, pp. 659-670, 1976.

[7] F. Camargo, A. Caminha, and H. de Lima, "Bernstein-type theorems in semi-Riemannian warped products," Proceedings of the American Mathematical Society, vol. 139, no. 5, pp. 1841-1850, 2011.

[8] H. F. de Lima, "Rigidity theorems in the hyperbolic space," Bulletin of the Korean Mathematical Society, vol. 50, no. 1, pp. 97103, 2013.

[9] L. J. Alías and A. G. Colares, "Uniqueness of spacelike hypersurfaces with constant higher order mean curvature in generalized Robertson-Walker spacetimes," Mathematical Proceedings of the Cambridge Philosophical Society, vol. 143, no. 3, pp. 703-729, 2007.

[10] Y. Wang and X. Liu, "On complete spacelike hypersurfaces in a semi-Riemannian warped product," Journal of Applied Mathematics, vol. 2013, Article ID 757041, 8 pages, 2013.

[11] L. J. Alías, A. Romero, and M. Sánchez, "Uniqueness of complete spacelike hypersurfaces of constant mean curvature in generalized Robertson-Walker spacetimes," General Relativity and Gravitation, vol. 27, no. 1, pp. 71-84, 1995.

[12] B. O'Neill, Semi-Riemannian Geometry with Applications to Relativity, Academic Press, New York, NY, USA, 1983.

[13] L. J. Alías, A. Romero, and M. Sánchez, "Spacelike hypersurfaces of constant mean curvature and Calabi-Bernstein type problems," The Tohoku Mathematical Journal, vol. 49, no. 3, pp. 337345, 1997.

[14] S. Montiel, "Unicity of constant mean curvature hypersurfaces in some Riemannian manifolds," Indiana University Mathematics Journal, vol. 48, no. 2, pp. 711-748, 1999.

[15] S. Montiel, "Uniqueness of spacelike hypersurfaces of constant mean curvature in foliated spacetimes," Mathematische Annalen, vol. 314, no. 3, pp. 529-553, 1999.

[16] C. P. Aquino and H. F. de Lima, "Uniqueness of complete hypersurfaces with bounded higher order mean curvatures in semiRiemannian warped products," Glasgow Mathematical Journal, vol. 54, no. 1, pp. 201-212, 2012.

[17] A. G. Colares and H. F. de Lima, "Some rigidity theorems in semi-Riemannian warped products," Kodai Mathematical Journal, vol. 35, no. 2, pp. 268-282, 2012.

[18] H. Bondi and T. Gold, "On the generation of magnetism by fluid motion," Royal Astronomical Society. Monthly Notices, vol. 108, pp. 252-270, 1948.

[19] F. Hoyle, "A new model for the expanding universe," Royal Astronomical Society. Monthly Notices, vol. 108, pp. 372-382, 1948.

[20] L. J. Alías and M. Dajczer, "Uniqueness of constant mean curvature surfaces properly immersed in a slab," Commentarii Mathematici Helvetici, vol. 81, no. 3, pp. 653-663, 2006. 


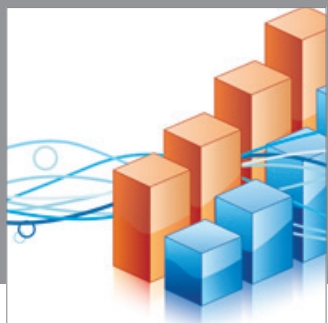

Advances in

Operations Research

mansans

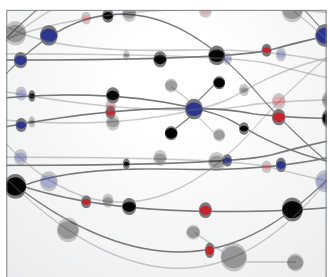

The Scientific World Journal
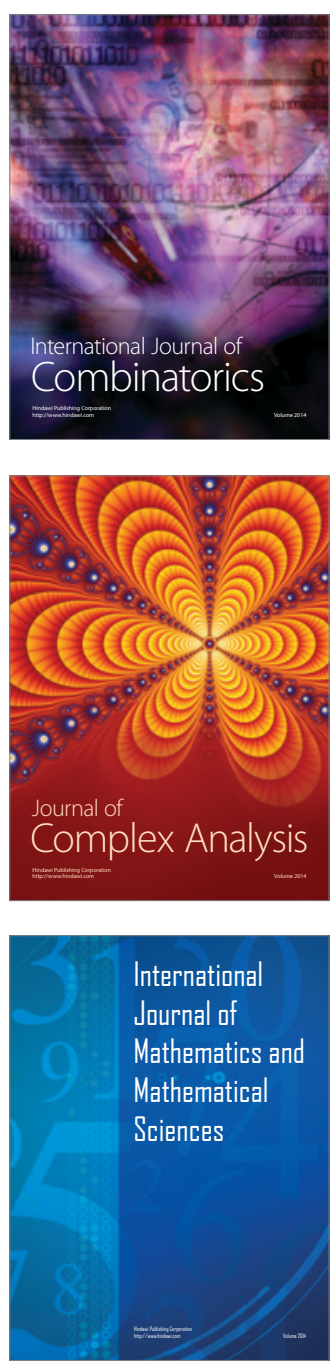
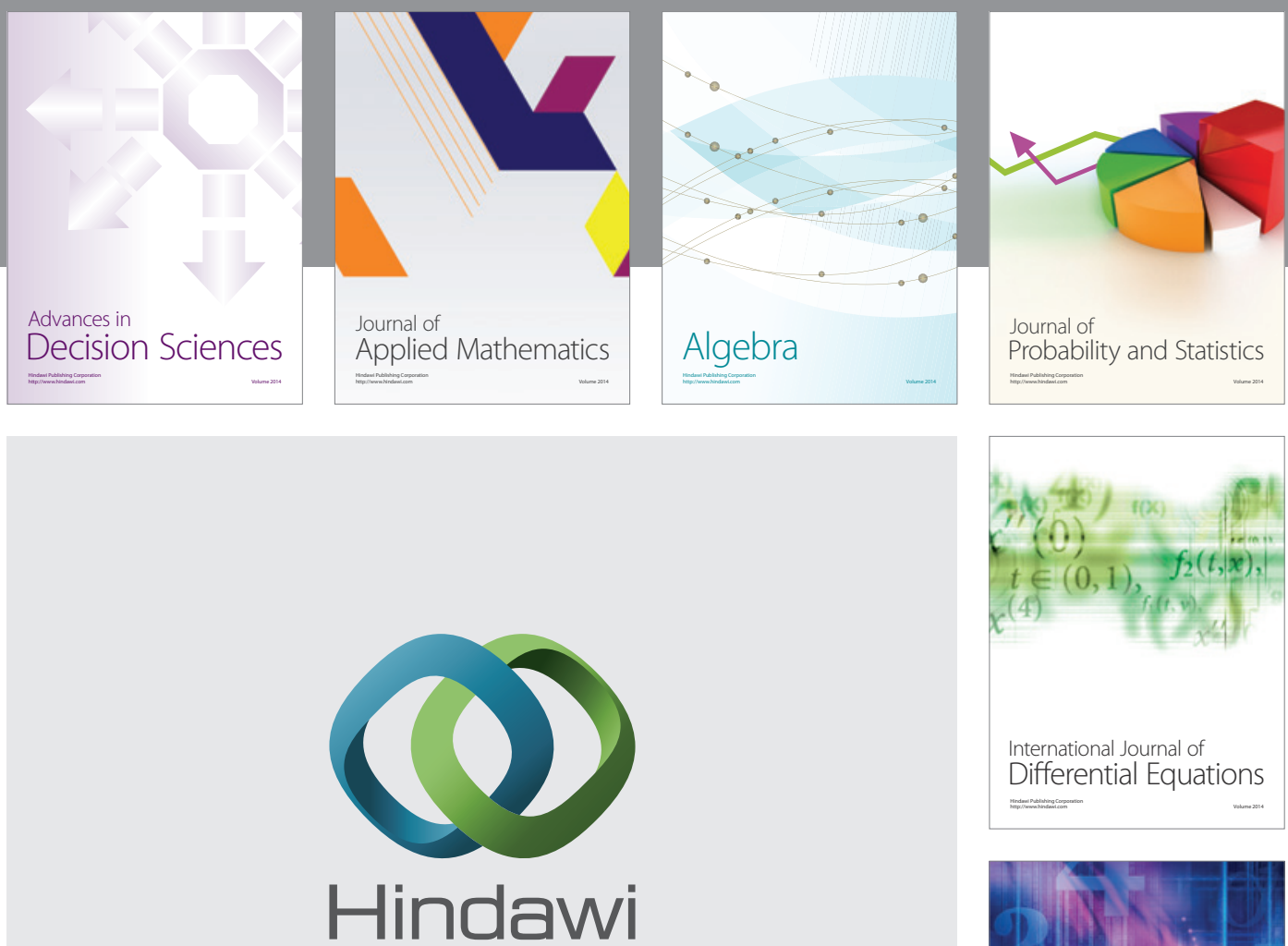

Submit your manuscripts at http://www.hindawi.com
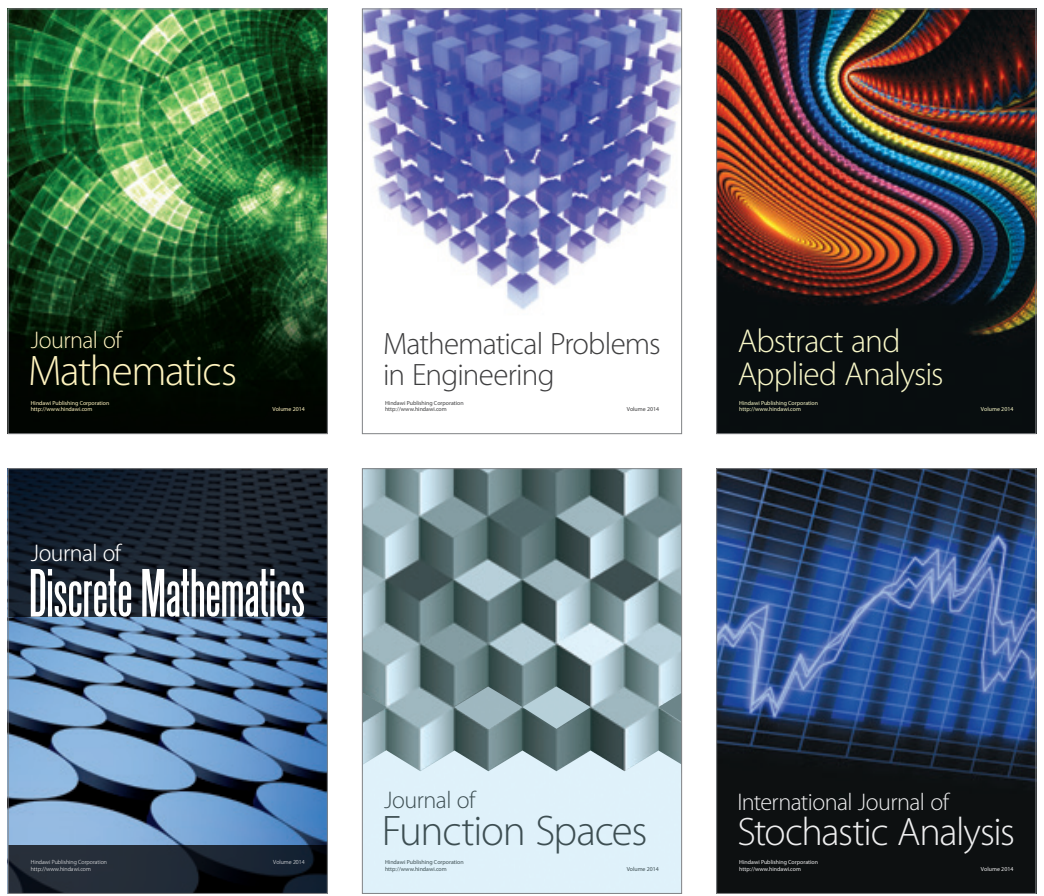

Journal of

Function Spaces

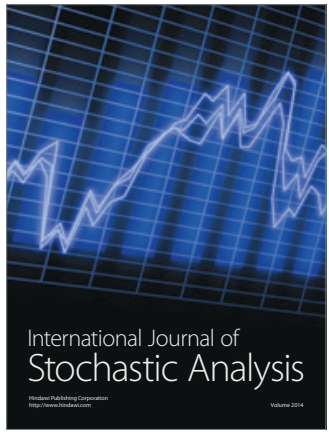

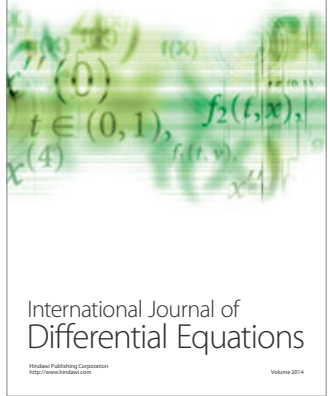
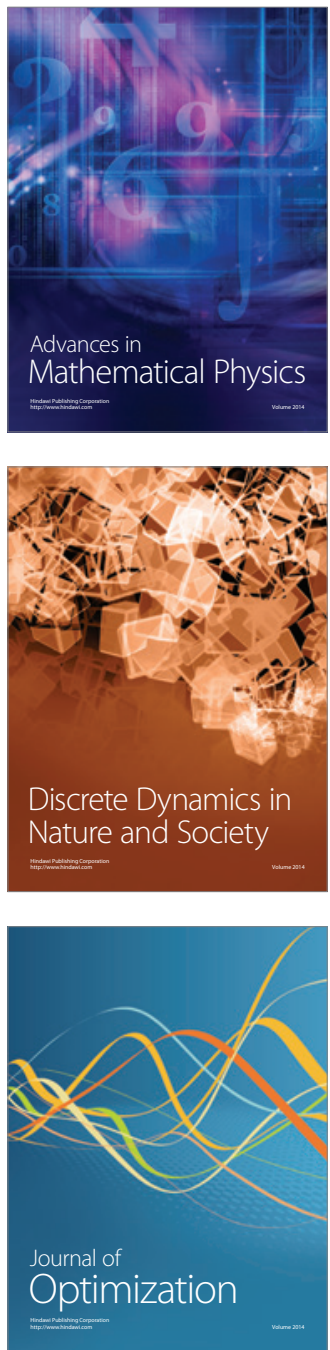